$$
\begin{aligned}
& \text { INviTED PAPER for Nonlineor Guided } w_{\text {aves }} \\
& \text { Mardn } 2508
\end{aligned}
$$

\title{
Nonlinear frequency conversion in two dimensional poled nonlinear crystals
}

\author{
N. G. R. Broderick, C. M. de Sterke,T. M. Monro and \\ D. J. Richardson \\ Optoelec tronics Research Centre, University of Southampton, Southampton, SO17 $1 B J, U K$ \\ C. M. de Sterke \\ Department of Theoretical Physics, University of Sydney, Sydney, Australia
}

We review our work on two dimensionally poled nonlinear materials. We have characterised the crystals at both high and low power and have started to model their behaviour. The results are compared with work using high power picosecond sources and future work is discussed.

The idea of quasi-phase matching (QPM) a nonlinear process by periodically inverting the nonlinearity has a long and venerable history since it was first suggested by Armstrong et al.. QPM allows highly dispersive materials which also have a high nonlinearity to be used in nonlinear experiments since the period of the nonlinear grating can be tailored to phase-match the desired nonlinear interaction. Originally the concept of QPM was restricted to one-dimensional geometries such as periodically poled lithium niobate (PPLN) but lately a number of researchers have looked at varying the nonlinearity in the transverse dimensions both aperiodically[1] and periodically[2]. Berger examined theoretically crystals whose nonlinearity varied periodically in two or three dimensions and called such structures nonlinear photonic crystals (NPC). In a NPC quasi-phase matching may be obtained in any direction where there is a suitable reciprocal lattice vector[2]. Recently we fabricated a sample of hexagonally poled lithium niobate (HeXLN)[3], which we believe is the first example of a 2D NPC, and observed non-collinear 2nd harmonic generation (SHG) using picosecond pulses with an efficiency as high as $80 \%$. In addition we examined the angular tuning of the HeXLN crystal and found it agreed well with theory.

Our original experiments were performed using a high power picosecond fibre laser at $1531 \mathrm{~nm}$ as the fundamental to pump the crystal. Due to the nature of the source we were unable to vary it's wavelength and so only the temperature and angle tuning of the SHG were measured. We observed a large temperature tuning bandwidth of $\sim 25^{\circ} \mathrm{C}$ for 2nd harmonic generation whereas a perfect PPLN crystal with the same length and period would have a bandwidth of $\sim 5^{\circ} \mathrm{C}$. The precise reason for this increased bandwidth is not known and analysis of the results is complicated by the bandwidth of the source, the large peak conversion efficiency and the walkoff between the pump and the 2nd harmonic. In order to obtain a better understanding of the mechanisms involved we have now performed similar measurements using a low power, (to ensure we are in the weak conversion limit) nanosecond wavelength tunable source on both a 2D HeXLN crystal and a 1D PPLN crystal with the same period and similar length. This allows us to directly compare the temperature and wavelength tuning curves and as shown here the curves for the $2 \mathrm{D}$ case are considerablely wider than the $1 \mathrm{D}$ case.

To describe the growth of the 2nd harmonic beam in a NPC one starts with the 2D version of Maxwell's equation with a nopnlinear polarisation. Then making the usual slowly varying envelope assumptions and for a linearly polarised input the growth of the 2nd harmonic beam is described by[2]

$$
\mathbf{k}^{2 \omega} \cdot \nabla E^{2 \omega}(\mathbf{r})=-2 i \frac{\omega^{2}}{c^{2}} \chi^{(2)}(\mathbf{r})\left(E^{\omega}\right)^{2} e^{\left(i\left(\mathbf{k}^{2 \omega}-2 \mathbf{k}^{\omega}\right) \cdot \mathbf{r}\right)}
$$

Since the nonlinear susceptibility tensor $\chi^{(2)}$ is periodic we can write it as a Fourier series:

$$
\chi^{(2)}(\mathbf{r})=\sum_{n, m} \kappa_{n, m} e^{i \mathbf{G}_{n, m} \cdot \mathbf{r}}, \quad n, m \in Z,
$$


where $\mathbf{G}_{n, m}$ are the reciprocal lattice vectors of the structure[4]. The phase matching condition,

$$
\mathbf{k}^{2 \omega}-2 \mathbf{k}^{\omega}-\mathbf{G}_{n, m}=0
$$

must be satisified for the interaction to proceed and determines the angle (or angles) at which the 2nd harmonic is emitted from the crystal.

The hexagonal structure of our 2D NPC is shown in Fig. 1. This structure was revealed by lightly etching the sample in acid. Each hexagon is a region of domain inverted material - the total inverted area comprises $25 \%$ of the overall sample area. The fabrication procedure was as follows. A thin layer of photoresist was first deposited onto the $-\mathrm{z}$ face of a $0.3 \mathrm{~mm}$ thick, z-cut wafer, of $\mathrm{LiNbO}_{3}$, and then photolithographically patterned with the hexagonal array. The $x-y$ orientation of the hexagonal structure was carefully aligned to coincide with the crystal's natural preferred domain wall orientation : $\mathrm{LiNbO}_{3}$ itself has trigonal atomic symmetry (crystal class $3 \mathrm{~m}$ ) and shows a tendency for domain walls to form parallel to the y-axis and at $\pm 60^{\circ}$ as seen in Fig. 1. Poling was accomplished by applying an electric field via liquid electrodes on the $+/$ z faces at room temperature[5]. Our HeXLN crystal has a period of $18.05 \mu \mathrm{m}$ : suitable for non-collinear frequency doubling of $1533 \mathrm{~nm}$ in the $\Gamma K$ direction at $140^{\circ} \mathrm{C}$ using the RLV lattice vector $G_{0,1}$ (an elevated temperature was chosen to eliminate any potential photorefractive effects). The hexagonal pattern was found to be uniform across the sample dimensions of $10 \times 7 \mathrm{~mm}(x-y)$ and was faithfully reproduced on the $+z$ face. Lastly we polished the $\pm x$-faces of the HeXLN crystal allowing a propagation length of $10 \mathrm{~mm}$ through the crystal in the $\Gamma K$ direction (see Fig. 1).
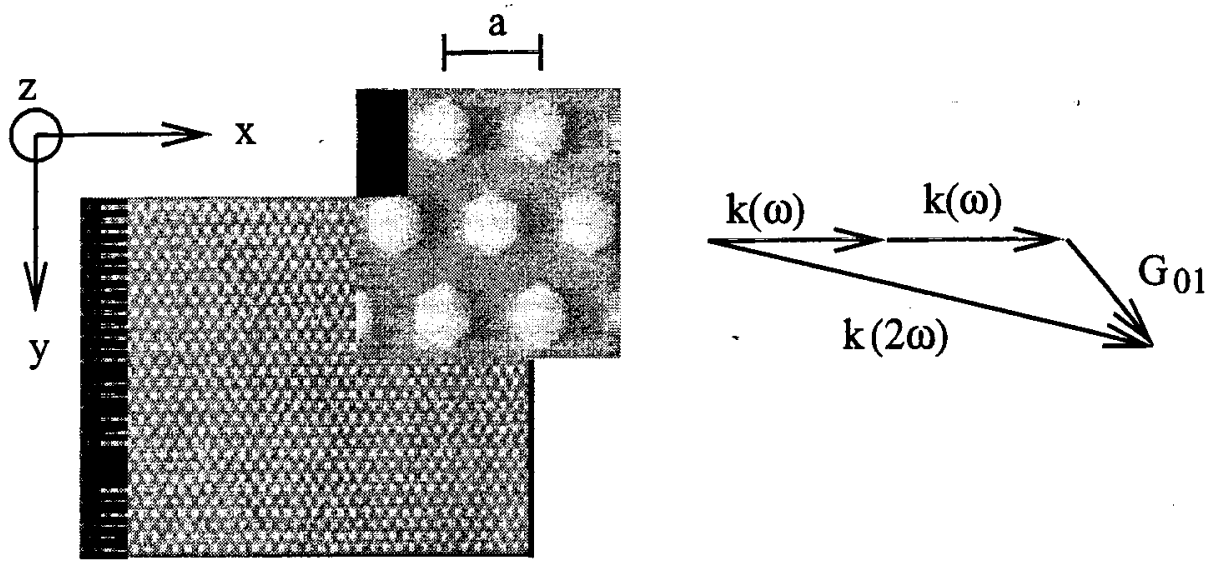

Fig. 1. Picture of the HeXLN crystal and wave vectors involved in the SHG process. The period $a$ of the crystal is $18.05 \mu \mathrm{m}$ is as shown in the picture and is uniform over the whole sample. In our experiments propagation was in the $+x$ direction.

To investigate the properties of the HeXLN crystal we proceeded as follows. The HeXLN crystal was placed in an oven and mounted on a rotation stage which could be rotated by $\pm 15^{\circ}$ around the z-axis while still allowing light to enter through the $+x$ face of the crystal. The fundamental pump beam consisted of $5 \mathrm{~ns}$, $1 \mathrm{~kW}$ transform limited square pulses obtained from a high power all-fibre cascaded amplification system operating at a pulse repetition rate of $10 \mathrm{kHz}$. The wavelength of the source could be varied continuously in the range $1520 \mathrm{~nm}-1560 \mathrm{~nm}$ and had a measured bandwidth of $<3 \mathrm{GHz}$ which was sufficient to resolve the spectral features of the crystal's response. The output from the amplifier was weakly focussed into the HeXLN crystal using a $17 \mathrm{~cm}$ focal length lens giving a focal spot diameter of $150 \mu \mathrm{m}$ (giving a depth of focus of $9 \mathrm{~mm}$ ) and a corresponding peak intensity of $\sim 0.5 \mathrm{MW} / \mathrm{cm}^{2}$. Note that zero angle of incidence corresponds to propagation along the $\Gamma K$ direction in the Brillouin zone while the closest RLVs are in the $\Gamma M$ directions. For these experiments the input angle was $\sim 4^{\circ}$ which was optimized for maximum 2 nd harmonic generation using $G_{0,1}[3]$. The output consisted of a 2 nd harmonic spot propagating at an angle of $\sim 2^{\circ}$ compared to the remaining fundamental. In addition there were multiple green spots corresponding to sum frequency generation between the fundamental and 2nd harmonic.

In addition to performing measurements on the HeXLN crystal we also used a $12 \mathrm{~mm}$ length of PPLN with a period of $18.05 \mu \mathrm{m}$ and a thickness of $0.5 \mathrm{~mm}$ for comparison. Fig. 2 shows the temperature response of the 

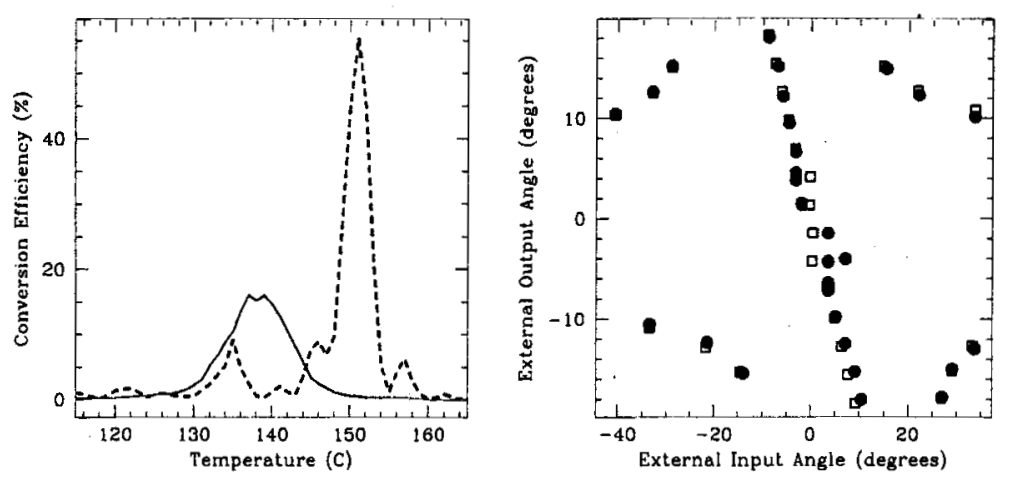

Fig. 2. The temperature dependence of the NPCs are shown above. Fig. $b$ shows the angular dependance of the 2 nd harmonic along with the theoretically predicted angles.

2nd harmonic external conversion efficiency for both the HeXLN (solid line) and the PPLN (dashed line) under identical experimental conditions. Note that as expected the PPLN crystal has a sinc ${ }^{2}$ shaped tuning curve and is quite narrow with a FWHM of $2.7^{\circ} \mathrm{C}$ as expected theoretically. In contrast the tuning curve for the HeXLN crystal is much broader with a FWHM of $9.5^{\circ} \mathrm{C}$ and has a more Gaussian profile. In addition the HeXLN crystal has a reduced efficiency as expected from the comparison of the Fourier coefficients discussed above. In Fig. 2 the two curves have a different centre wavelength. This is due to the angle of incidence of the fundamental light into the HeXLN crystal. By angle tuning the crystal phase-matching can be achieved at any temperature between 100 and 200 degrees with the same efficiency. The effect of angle tuning the crystal at a fixed temperature is shown in Fig. $2 \mathrm{~b}$ which shows the output 2nd harmonic angle along with the associated RLV for a range of input angles.

In addition we have begun some numerical modelling of Eq. 1 for the HeXLN crystal. Our approach is based on a Green function analysis and will be described more fully elsewhere. We find that there is excellent agreement between the theoretical and experimental results. In particular the theoretical curve reproduces the line shape of the HeXLN response and is non-sinc like. This non-sinc like response is due to the noncollinear nature of the interaction in the HeXLN crystal and could just as easily be observed by angle tuning the PPLN crystal. Similarly the broader bandwidth of the crystal is due to the extra degree of freedom in a 2D geometry for phase-matching and at different temperatures the 2nd harmonic light is emmitted from the crystal at slightly different angles (the angular spread is typically less than 2 degrees) which allows the phase matching criterion to be maintained.

These results presented here show that due to the noncollinear nature of the 2nd harmonic generation process in $\mathrm{H}_{\mathrm{e}} \mathrm{XLN}$ the bandwidth is considerable larger than a comparable collinear interaction in a PPLN crystal. The measured bandwidth of our crystal is in excellent agreement with the results of our numerical model. The shape of the wavelength response is critically dependant on the incident angle as expected. In addition the major advantage of such crystals will be in phase-matching multiple nonlinear interactions which is not possible in general in a PPLN crystal.

\section{References}

1. G. Imeshev, M. Proctor, and M. M. Fejer, "Lateral patterning of nonlinear frequency conversion with transversely varying quasi-phase-matching gratings," Opt. Lett. 23, 673-675 (1998).

2. V. Berger, "Nonlinear Photonic Crystals," Phys. Rev. Lett. 81, 4136-4139 (1998).

3. N. G. R. Broderick, H. L. Offerhaus, G. W. Ross, D. J. Richardson, and D. C. Hanna, "HeXLN: A 2-Dimensional nonlinear periodic crystal," Phys. Rev. Lett. , ( ).

4. C. Kittel, Introduction to Solid State Physics, 3rd ed. (John Wiley and Sons, New York, 1953).

5. M. Yamada, N. Nada, M. Saitoh, and K. Watanabe, "1st-order Quasi-phase matched LINBO3 wave-guide periodically poled by applying an external-field for efficient blue 2nd-harmonic generation," Appl. Phys. Lett. 62, 435-436 (1993). 